\title{
3D Reconstruction, Modelling and Analysis of in Situ Root System Architecture
}

\author{
Pankaj Kumar, Jinhai Cai , Stan Miklavcic \\ Phenomics and Bioinformatics Research Centre (PBRC) School of Information Technology and \\ Mathematical Sciences, University of South Australia, Mawson Lakes, SA 5095 \\ Australian Centre for Plant Functional Genomics (ACPFG), University of Adelaide, Urbane SA, 5064 .
}

Email: pankaj.kumar@unisa.edu.au

\begin{abstract}
Root system architecture (RSA) plays an important role in water and nutrient uptake for plant development and growth and hence in grain yield. In situ studies of RSA root architecture will assist in the characterization of phenotypes for the purpose of identifying genotypes of cereal plants that are more stress tolerant and are producer of higher grain yield. In this paper we present a method of 3D reconstruction of roots with a non-destructive method, preserving the RSA and also allowing for observation of the roots growth as function of time.

Our method was applied to corn plant seedlings in their early stage of growth. The germinated corn seeds are grown in a transparent gel-based growth system. Observations are made with a digital camera with the growth chamber sitting on a turntable, at different time instances of the growth. The digital images are processed for segmentation of roots, root tip detection, and root tips are tracked as a function of angle of the turntable motion. The elliptical trajectories of the root tips are used to calibrate the cameras. After calibration of the imaging sensor the 3D reconstruction and modelling of the roots are done using the visual hull (space carving) algorithm. We analyse different stages of 3D root developments in terms of roots volume as a function of time and the number of images used in reconstruction of the RSA.

Our results show that root biomass/volume increases with time. This trend is true irrespective of the number of images used for 3D reconstruction. The increase in root volume is approximately linear with time for plants is in the early stage of growth. It is not very clear what will be the optimal number of images to use in $3 \mathrm{D}$ reconstruction and modelling. Less number of images require less acquisition time and less processing time, which may be important for high throughput systems. By visual inspection it can be said that the $3 \mathrm{D}$ reconstruction becomes more accurate and detailed with increasing number of images. We empirically verify that the volume of reconstruction monotonically decreases increasing number of images from different viewpoints. This is due to erroneous voxels being carved out when information from more images are being added to $3 \mathrm{D}$ reconstruction.
\end{abstract}

Keywords: 3D Reconstruction, Root System Architecture, Temporal Growth Analysis, Gellan gum 


\section{INTRODUCTION}

Root system architecture (RSA) is a fundamental trait related to agricultural and natural ecosystem productivity Lynch (1995); Hammer et al. (2009); Hodge et al. (2009); De Smet et al. (2012). A cereal plant's yield is greatly influenced by the plants ability to adapt its RSA to the environment. Several researchers Moreno-Risueno et al. (2010); Traas and Vernoux (2010) highlight the role of genes in regulating root growth rate and branching patterns. Thus, automated 3D reconstruction of the RSA and from this root biomass estimates, applied across genetic varieties, have the potential of being an important plant physiological tools. Several approaches using image processing and 3D reconstruction for the quantitative analysis of RSA have been developed, Lobet et al. (2011); Clark et al. (2011); Zheng et al. (2011). Lobet et al. (2011) proposed a semi-automated image processing technique to streamline the quantitative analysis of growth and structure development of complex root systems. Their software, SmartRoot, is an operating system-independent freeware, based on ImageJ and relies on cross platform standards for communication with data-analysis. Clark et al. (2011) developed a high-throughput phenotyping method for the tracking of 3D root traits during seedling development. Their platform has high flexibility and capacity to measure root traits at high spatial and temporal resolution. However, most of the root features are manually selected by the user and are thus semi-automated at best . Zheng et al. (2011) presented a scheme for high resolution, 3D root reconstruction using the concept of a visual hull. Apart from the use of a visual sensor, other methods have been developed to obtain the RSA of a plant. Kutschera (1960) used manual drawing to visualize RSA of a wide range of plant species, ranging from Barley plants to Pine trees. Manual drawings are a good way to fuse human observation with field and experimental observations. Esser et al. (2010) used neutron radiography (2-D) and tomography (3-D) to study in situ water distributions and root structure uptake of water in lupin and maize. Mairhofer et al. (2012) used x-ray microcomputed tomography to reconstruct the 3D RSA of root, in situ in carefully prepared soil environment. Lidar was used for 3D reconstruction of root and was applied to crop phosphorus research in Fang et al. (2009). Magnetic resonance imaging for the study of RSA and its different functional aspects were done by Stingaciu et al. (2012); Uwe et al. (2011) and Pohlmeier et al. (2008).

In this paper we report our complete system developed for the 3D reconstruction and modelling of RSA for roots grown in a transparent growth medium. The system presented here is a part of an automatic system for root phenotyping. Visual sensor cameras are used to capture a sequence of images of the roots as a function of rotation angle of the turntable. The turn table undergoes a single axis motion. A triangulated surface mesh model is generated from the 3D reconstruction volume to model the roots. The method developed is analysed in terms of the number images in the turntable sequence that are needed for the 3D reconstruction of the RSA and the volume of the root as a function of time.

The rest of the paper is organized as follows: In Section 2 we give the modular design of our system and briefly state the function of each modules. Segmentation, root tip detection and tracking, 3D reconstruction are discussed in Sections 3, 4, and 5, respectively. Results are discussed and analysed in Section 6. Finally, we conclude the paper and discuss the ideas for future work in Section 7.

\section{SySTEM Design}

We use a combination of preprocessing for segmentation/silhouette generation, feature detection, semantic feature identification by supervised statistical learning, tracking, self-calibration and mesh modelling. With this combination an automated RSA reconstruction and modelling system is developed. The sequential modules are:

1. Root images are segmented and silhouettes of each image is generated using algorithms described in Cai and Miklavcic (2013)

2. High curvature features are detected as described in He and Yung (2008) and a regional feature descriptor of an image patch $64 \times 64$ around high curvature point is computed using Zernike moments (Kumar et al. (2012b)) .

3. The above detected feature points are classified into semantically meaningful feature points of being root tip or non-root tip using the method described in (Kumar et al. (2012a)).

4. The root tips detected in each image of the sequence are tracked as a function of rotation angle and an ellipse is fitted to the trajectory of the root tips.

5. The conics thus generated are used to obtain the parameters of the imaging setup using the method described in Jiang et al. (2002). 
Kumar et al., 3D Reconstruction, Modelling and Analysis of in Situ Root System Architecture

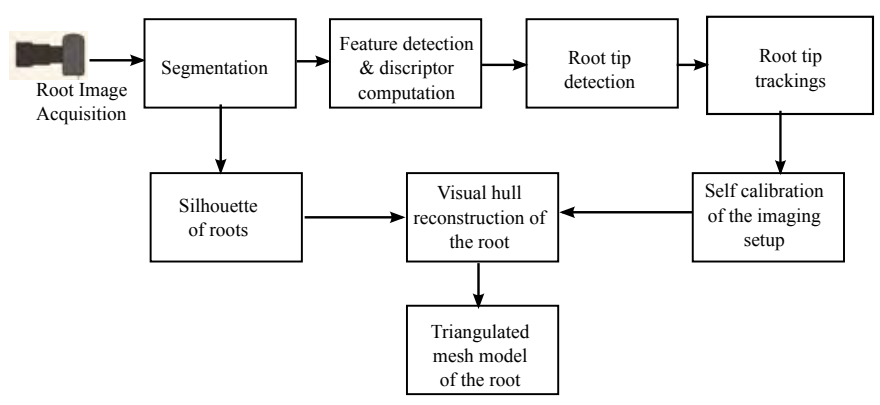

Figure 1. Block diagram schematic of the proposed root 3D reconstruction and modelling system.

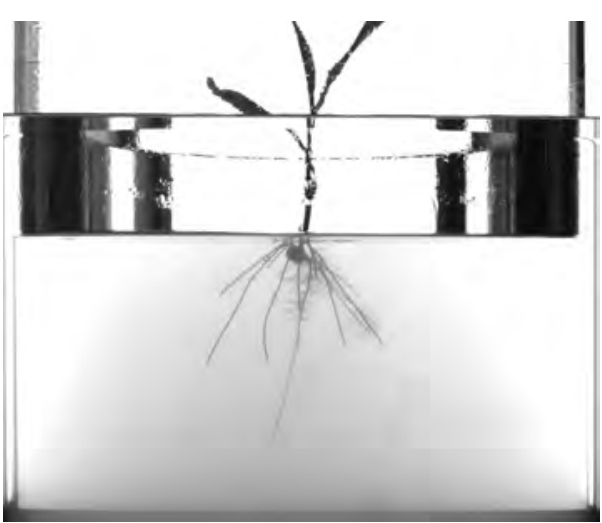

(a)

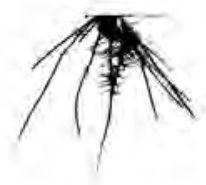

(b)

Figure 2. (a) is an image of 18 days old corn root at $90^{\circ}$ rotation on the turn table and (b) is segmented root silhouette obtained for the image and used in our implementation.

6. Visual hull reconstruction of the roots is carried out using the results of root silhouettes and the parameters of the imaging setup.

7. $3 \mathrm{D}$ voxel reconstruction is modelled into a triangulated polygonal mesh model.

Figure 1 shows the schematic of the system design.

\section{SEgMENTATION AND ROOT SILHOUETTE}

For segmentation of the root from its background gellan gum medium we have used the method described in Cai and Miklavcic (2013). In this method the background gellan gum with its refracted distribution of light is modelled as a high-order parametric surface. The local intensity is a function of the pixel location. The parameters of the surface are obtained by sampling the image at different points in the grid using the RANSAC Fischler and Bolles (1981) algorithm. Figure 2(a) shows a raw corn root image; its segmented silhouette is shown in Figure 2(b). When using this silhouette for 3D reconstruction we invert the bi-level image of Figure 2(b).

\section{ROOT TIP DETECTION AND TRACKING}

We have used Zernike moments for representation and detection of root tips. The reason for using Zernike moments are its nice properties of orthogonality, rotation invariance and demonstrated effective application to bi-level images Khotanzad and Hong (1990); Teague (1980). Orthogonality of Zernike moments validates the assumption of independence of the moments. Hence, in Gaussian mixture modelling (GMM) we can fix the covariance matrix to be diagonal which significantly decreases the computation time thus achieving real time processing. For the purpose of classifying an image patch $I^{c}(x, y)$ or testing the classification algorithm, the magnitudes of the $n^{t h}$ order Zernike moments are computed and then, using Bayes rule, the density of class root tip given the Zernike moments is computed. In our results we achieve as high as $100 \%$ true detection 


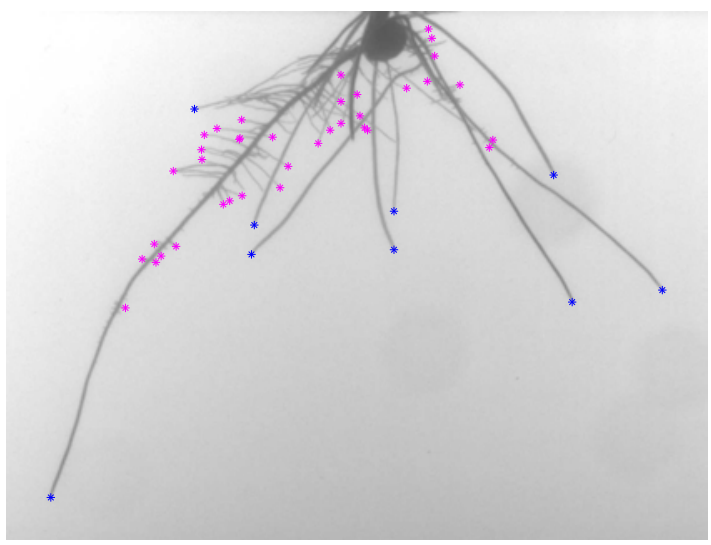

(a)

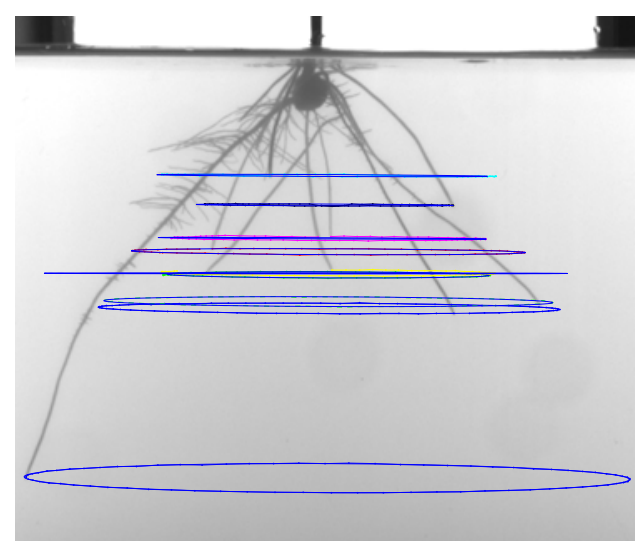

(b)

Figure 3. (a) is an image of 18 days old corn root on which primary root tip detection are show in blue asterisk and lateral root tip detections are shown in cyan asterisk. (b) is the tracking of the primary root tips in the 72 images of the image sequence and fitting of ellipse to these trajectories.

rate at the cost of less than $2.5 \%$ false alarm rate. The readers are referred to Kumar et al. (2012a) for details of this implementation. Figure 3(a) shows an example of root tip detection. Figure 3(b) shows the tracking of these root tips across the 72 image sequence obtained from the turntable rotation. For tracking of the root tips we have used the method of multi-target tracking described in Kumar et al. (2006). The method cooperatively combines Kalman filter-based motion and region tracking with an efficient geometric shape matching algorithm. The system is fully automated requiring no manual input of any kind for initialization of tracking. The target track initialization problem is formulated as computation of shortest paths in a directed and attributed graph using Dijkstra's shortest path algorithm. This scheme correctly initializes multiple target tracks for tracking even in the presence of clutter and segmentation errors which may occur in detecting a target. For more details of the method of tracking please refer to Kumar et al. (2006). To make the tracking problem simple and fast we have tracked only the seminal roots and not the lateral roots. Once the trajectory of the seminal root tips has been obtained an ellipse fitting is applied to the trajectory points using the algorithm described in Kumar et al. (2013).

\section{3D RECONSTRUCTION USING VISUAL HULL METHOD}

Visual hull method is multiple view method of 3D volumetric reconstruction of the object being imaged. Object's silhouettes are used to reconstruct the object by finding the common intersection of visual cones formed by the object's silhouettes and the camera centres. The silhouettes determine whether each voxel in the total volume belongs to the object or not by projecting a line-of-sight ray from the camera centre. This approach to volume reconstruction was developed by Laurentini (1995). For our 3D reconstruction we have used the method described by Forbes et al. (2003) and the codes provided by them. There are other methods of fast 3D reconstruction Yous et al. (2007), which can be applied is camera parameters are known. After 3D reconstruction the voxel representation of the plant is converted to a polygonal mesh model.

\section{Results}

In results we show the 3D reconstruction and modelling of a corn root at its different stages of development and also when different number of images are used for reconstruction of the root. Figure 4 . shows the reconstruction of the RSA at: day 6 - (a), day 10 - (b), day 14 - (c), and day 18 - (d). These reconstructions are sufficiently detailed and truly portrays the complex 3D structure of RSA. Models in these images have been developed using all 72 images from the turntable image sequence. Figure 5. shows the reconstruction of the root with different number of images used for 3D reconstruction: 12 images - (a), 24 images - (b), 36 images - (c), 48 images - (d) , 60 images - (e) and 72 images - (f). The results are when the plant is at day $14^{\text {th }}$ of its growth. There are subtle differences in these 3D models of the root. As the number of images in the 3D reconstruction are increased the fine structures of the RSA are captured and represented in the 3D model. This can be noted by observing the longest seminal root in these models. This seminal root is longest and more vividly captured in Figure 5 (f) than the other images. The stoutness of the models from Figure 5 (a)-(f) seems 


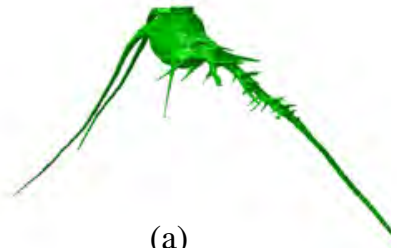

(a)

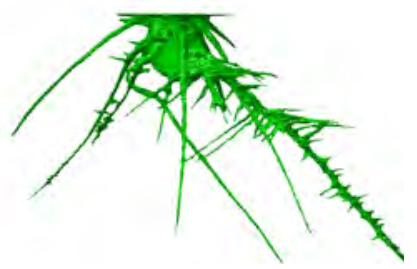

(c)
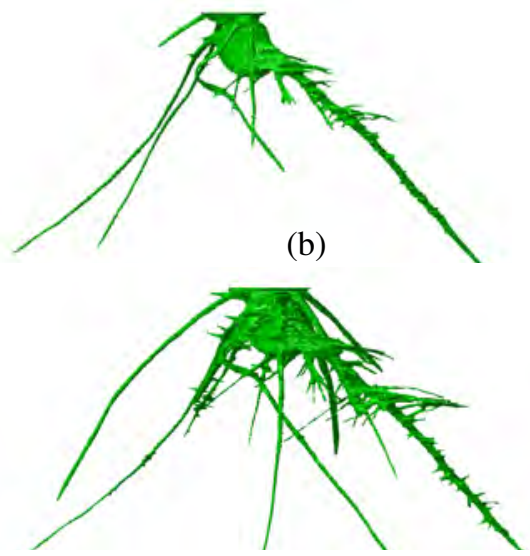

(d)

Figure 4. (a, b, c, d) are the results of 3D reconstruction for day 6,10,14, 18 of the growth of corn roots The results by the proposed method are detailed enough for phenotyping analysis

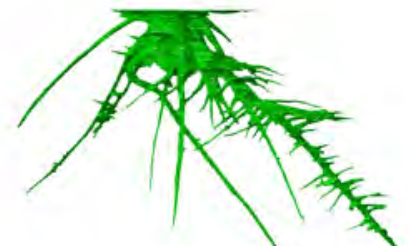

(a)

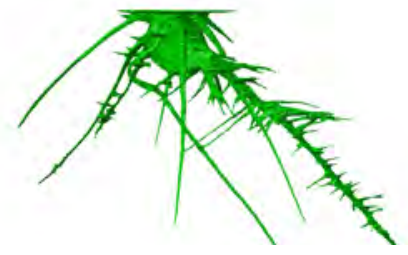

(d)

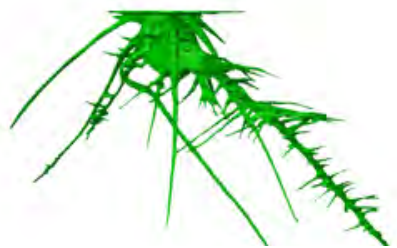

(b)

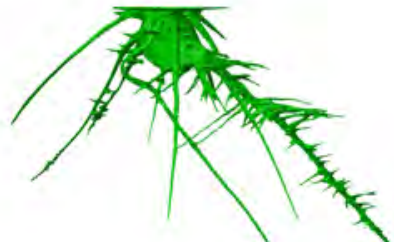

(e)

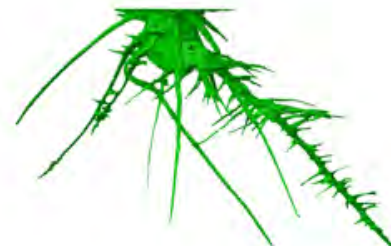

(c)

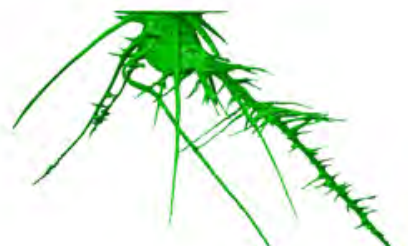

(f)

Figure 5. (a, b, c, d,e, f ) are the results of $3 \mathrm{D}$ reconstruction with 12, 24, 36, 48, 60, 72 number of images respectively, from the turntable sequence. The images are from the day 14 growth stage of the corn plant.

to decrease, this is observation is similar to that of plot 6 (b), where the volume of the RSA decreases with increase in the number of images for $3 \mathrm{D}$ reconstruction.

Figure 6 shows the plot of RSA volume as a function of measurement day 6(a) and as a function of number of images used in 3D reconstruction 6(b). The root growth is almost linear with time as the plant is its early stage of growth. However, the RSA volume as a function of the number of images goes down with increase in the number of images for 3D reconstruction. This phenomena can be explained by the fact that as we increase the number of images the carving by visual hull method becomes more precise and more erroneous voxels are removed with increase in the number of images. When more images are used for $3 \mathrm{D}$ reconstruction then the 3D model becomes more accurate to the RSA however the time for processing increases.

\section{CONCLUSIONS}

In this paper we have presented a complete system for 3D reconstruction and modelling of roots grown in transparent gellan gum medium. Although not a natural environment for growing plants, never the less it represents a useful strategy for phenotyping roots and studying the 3D structure using relatively in-expensive techniques. Visual sensors (digital) cameras are much cheaper to procure, operate, and maintain than 3D CT X-ray scanners, MRI, or even Lidar. We have demonstrated the successful working of the proposed method 
Kumar et al., 3D Reconstruction, Modelling and Analysis of in Situ Root System Architecture

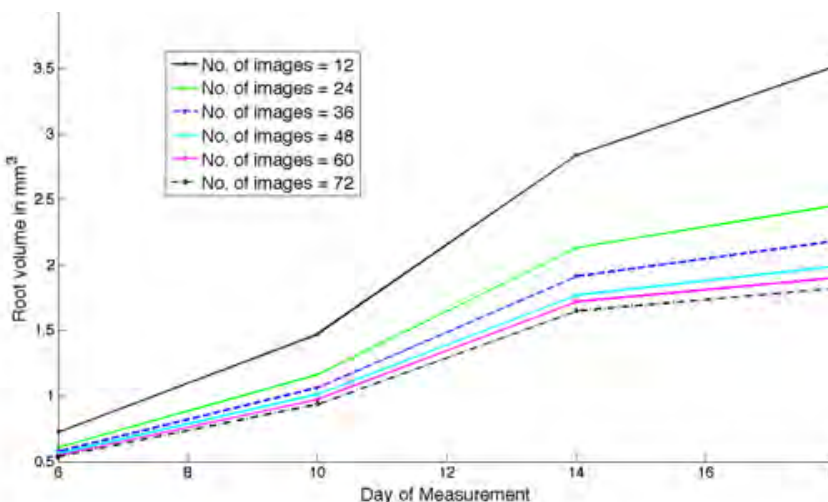

(a)

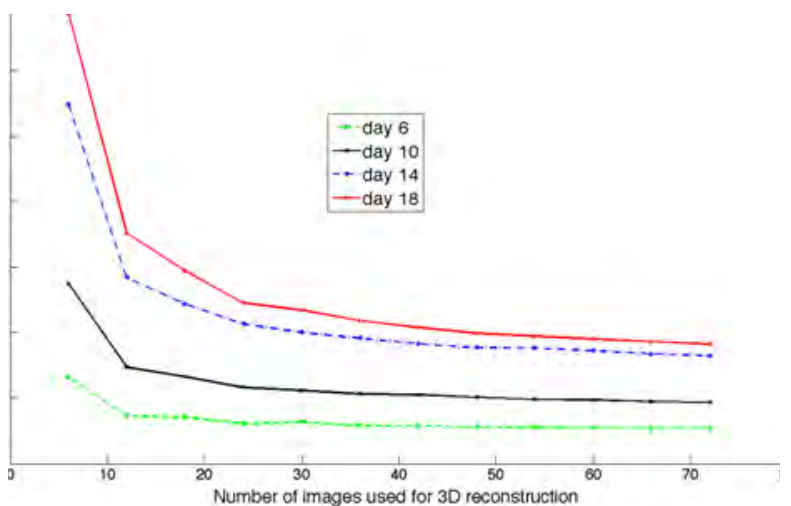

(b)

Figure 6. (a) is a plot of corn roots 3D volume as a function of the day of measurement. This plot is very close to being linear as the plant is in its early stage of growth. (b) is a plot of root volume as a function of number of images used in 3D reconstruction of the root. The measured volume of RSA decreases with increasing number of images, however the difference in volume/biomass at different days of growth is consistent.

on corn roots. Detailed reconstruction of the roots where obtained with 72 images. The root growth rate is observed to be almost linear with time, which is well in line with current theories for plants in biology that is a plant in its early stage of growth has almost a linear growth rate. Increasing the number of images for 3D reconstruction improves the accuracy of 3D model although it decreases the observed volume. This empirical finding is consistent with the theory of space carving which states that the volume of reconstruction decreases monotonically with increasing number of images from different view points. In our future experiments we will also consider the effect of changing viewing angles. We will also report on the issues due to refraction of light through gellan gum and its impact on 3D reconstruction. The root shape can be analysed using statistical shape analysis techniques like Kurtek et al. (2013).

\section{ACKNOWLEDGEMENT}

The authors would like to thank the financial support from the Australian Research Council(ARC) and the South Australian State Goverment. Authors are grateful to Dr. Hamid Laga for thoughtful discussions on camera calibration and $3 \mathrm{D}$ reconstruction.

\section{REFERENCES}

Cai, J. and S. Miklavcic (2013). Surface fitting for individual image thresholding and beyond. IET Image Processing To appear.

Clark, R. T., R. B. MacCurdy, J. K. Jung, J. E. Shaff, S. R. McCouch, D. J. Aneshansley, and L. V. Kochian (2011). Three-dimensional root phenotyping with a novel imaging and software platform. Plant Physiology 156(2), 455-465.

De Smet, I., P. J. White, A. G. Bengough, L. Dupuy, B. Parizot, I. Casimiro, R. Heidstra, M. Laskowski, M. Lepetit, F. Hochholdinger, X. Draye, H. Zhang, M. R. Broadley, B. Pret, J. P. Hammond, H. Fukaki, S. Mooney, J. P. Lynch, P. Nacry, U. Schurr, L. Laplaze, P. Benfey, T. Beeckman, and M. Bennett (2012). Analyzing lateral root development: How to move forward. The Plant Cell 24(1), $15-20$.

Esser, H. G., A. Carminati, P. Vontobel, E. H. Lehmann, and S. E. Oswald (2010). Neutron radiography and tomography of water distribution in the root zone. Journal of Plant Nutrition and Soil Science 173(5), 757-764.

Fang, S., X. Yan, and H. Liao (2009). 3d reconstruction and dynamic modeling of root architecture in situ and its application to crop phosphorus research. The Plant Journal 60(6), 1096-1108.

Fischler, M. A. and R. C. Bolles (1981, June). Random sample consensus: a paradigm for model fitting with applications to image analysis and automated cartography. Commun. ACM 24(6), 381-395.

Forbes, K., F. Nicolls, G. Jager, and A. Voigt (2003). Using silhouette consistency constraints to build 3d models. In Proceed-ings of the Fourteenth Annual South African Workshop on Pattern Recognition, Lecture Notes in Computer Science.

Hammer, G., Z. Dong, G. Mclean, A. Doherty, C. Messina, J. Schussler, C. Zinselmeier, S. Paszkiewicz, and M. Cooper (2009). Can changes in canopy and/or root system architecture explain historical maize yield trends in the U.S. corn belt? Crop Science 49, 299-312.

He, X. C. and N. H. C. Yung (2008, May). Corner detector based on global and local curvature properties. Optical Engineering 47(5). 
Kumar et al., 3D Reconstruction, Modelling and Analysis of in Situ Root System Architecture

Hodge, A., G. Berta, C. Doussan, F. Merchan, and M. Crespi (2009, May). Plant root growth, architecture and function. Plant Soil 321(12), 153-187.

Jiang, G., H.-t. Tsui, L. Quan, and A. Zisserman (2002). Single axis geometry by fitting conics. In A. Heyden, G. Sparr, M. Nielsen, and P. Johansen (Eds.), Computer Vision ECCV 2002, Volume 2350 of Lecture Notes in Computer Science, pp. 537-550. Springer Berlin Heidelberg.

Khotanzad, A. and Y. H. Hong (1990, May). Invariant image recognition by zernike moments. IEEE Transactions on Pattern Analysis and Machine Intelliegence 12, 489-497.

Kumar, P., J. Cai, and S. Miklavcic (2012a). Automated detection of root crowns using gaussian mixture model and bayes classification. In DICTA, pp. 1-7.

Kumar, P., J. Cai, and S. Miklavcic (2012b). Root crown detection using statistics of zernike moments. In Control Automation Robotics Vision (ICARCV), 2012 12th International Conference on, pp. 1130-1135.

Kumar, P., J. Cai, and S. Miklavcic (2013). Improved ellipse fitting by considering the eccentricity of data point sets. In ICIP, pp. To appear.

Kumar, P., S. Ranganath, K. Sengupta, and H. Weimin (2006). Cooperative multitarget tracking with efficient split and merge handling. Circuits and Systems for Video Technology, IEEE Transactions on 16(12), 1477-1490.

Kurtek, S., A. Srivastava, E. Klassen, and H. Laga (2013). Landmark-guided elastic shape analysis of spherically-parameterized surfaces. Computer Graphics Forum 32(2pt4), 429-438.

Kutschera, L. (1960). Wurzelatlas mitteleuropischer ackerunkruter und kulturpflanzen. DLG, Frankfurt am Main.

Laurentini, A. (1995). How far 3d shapes can be understood from $2 \mathrm{~d}$ silhouettes. Pattern Analysis and Machine Intelligence, IEEE Transactions on 17(2), 188-195.

Lobet, G., L. Pags, and X. Draye (2011). A novel image-analysis toolbox enabling quantitative analysis of root system architecture. Plant Physiology 157(1), 29-39.

Lynch, J. (1995). Root architecture and plant productivity. Plant Physiology 109, 7-13.

Mairhofer, S., S. Zappala, S. R. Tracy, C. Sturrock, M. Bennett, S. J. Mooney, and T. Pridmore (2012). Rootrak: Automated recovery of three-dimensional plant root architecture in soil from x-ray microcomputed tomography images using visual tracking. Plant Physiology 158(5), 561-569.

Moreno-Risueno, M. A., J. M. Van Norman, A. Moreno, J. Zhang, S. E. Ahnert, and P. N. Benfey (2010). Oscillating gene expression determines competence for periodic arabidopsis root branching. Science 329(5997), 1306-1311.

Pohlmeier, A., A. Oros-Peusquensb, M. Javauxad, M. I. Menzelc, J. Vanderborghta, J. Kaffankeb, S. Romanzettib, J. Lindenmairc, H. Vereeckena, and N. J. Shahb (2008). Changes in soil water content resulting from ricinus root uptake monitored by magnetic resonance imaging. Vadase Zone Journal 7(3), 1010-1017.

Stingaciu, L., H. Schulz, A. Pohlmeier, S. Behnke, H. Zilken, M. Javaux, and H. Vereecken (2012). In situ root system architecture extraction from magnetic resonance imaging for water uptake modeling. Vadase Zone Journal 12(1), on-line access.

Teague, M. R. (1980). Image analysis via the general theroy of moments. Journal of Optical Society of America 70(8), 920-930.

Traas, J. and T. Vernoux (2010). Oscillating roots. Science 329(5997), 1290-1291.

Uwe, R., B. Stephan, F. Fabio, J. Siegfried, J. Marcus, K. A. J., M. Shizue, M. L. L. A., M. Andrew, M. Ralf, M.-L. Mark, N. K. A., P. Roland, P. Francisco, S. C. M., T. V. M., T. M. R., van Dusschoten Dagmar, van Volkenburgh Elizabeth, W. C. W., and S. Ulrich (2011). Non-invasive approaches for phenotyping of enhanced performance traits in bean. Functional Plant Biology $38,968983$.

Yous, S., H. Laga, M. Kidode, and K. Chihara (2007). Gpu-based shape from silhouettes. In Proceedings of the 5th international conference on Computer graphics and interactive techniques in Australia and Southeast Asia, pp. 71-77. ACM.

Zheng, Y., S. Gu, H. Edelsbrunner, C. Tomasi, and P. Benfey (2011, November). Detailed reconstruction of 3d plant root shape. In IEEE International Conference on Computer Vision, pp. 2026-2033. 